\title{
Optimization of intuitionistic and type-2 fuzzy systems in control
}

\author{
Oscar Castillo \\ Tijuana Institute of Technology \\ Division of Graduate Studies and Research \\ Calzada Tecnologico s/n, Fracc. Tomas Aquino \\ 22379 Tijuana, Mexico \\ e-mail: ocastillo@tectijuana.mx
}

\begin{abstract}
In this paper a method for finding the optimal design of intuitionistic fuzzy systems in control applications is presented. Traditional models work with type-0, which means using precise numbers in the models, but since the seminal work of Prof. Zadeh in 1965, type-1 fuzzy models emerged as a powerful way to represent human knowledge and natural phenomena. Later type-2 fuzzy models were also proposed by Prof. Zadeh in 1975 and more recently have been studied and applied in real world problems by many researchers. In addition, as another extension of type-1 fuzzy logic, Prof. Atanassov proposed Intuitionistic Fuzzy Logic, which is a very powerful theory in its own right. Previous works of the author and other researchers have shown that certain problems can be appropriately solved by using type- 1 , and others by interval type-2, while others by using intuitionistic fuzzy logic. Bio-inspired and meta-heuristic optimization algorithms have been commonly used to find the optimal design of type-1, type- 2 or intuitionistic fuzzy models for applications in control, robotics, pattern recognition, time series prediction, just to mention a few. However, the question still remains about if even more complex problems (meaning non-linearity, noisy, dynamic environments, etc.) may require even higher types, orders or extensions of type-1 fuzzy models to obtain better solutions to real world problems. In this paper a framework for solving this problem of finding the optimal fuzzy model for a particular problem is presented. To the knowledge of the author, this is the first work to propose a systematic approach to solve this problem, and we envision that in the future this approach will serve as a basis for more efficient algorithms for the same task of finding the optimal fuzzy system.
\end{abstract}

Keywords: Intuitionistic fuzzy systems, Type-2 fuzzy systems, Type-1 fuzzy systems.

2010 Mathematics Subject Classification: 03E72. 


\section{Introduction}

Nowadays it is well accepted that either type-1, type- 2 or intuitionistic fuzzy systems can solve many real world problems. Initially, type-1 fuzzy logic was proposed and applied in a plethora of real world problems ranging from control, to pattern recognition and time series prediction $[8,10,12]$. Although, more recently it has been recognized that type-1 fuzzy sets do not really handle uncertainty, because they only use precise values for the membership values. For this reason, type-2 fuzzy logic emerged as an extension of its type-1 counterpart. Type- 2 fuzzy systems use membership functions whose values are fuzzy sets [7, 11, 13]. Of course, as a special in between case, we have interval type- 2 fuzzy systems that use membership functions whose values are intervals. In the literature, we can find many cases in which type- 1 fuzzy systems have adequately solve real world problems. However, for more complex situations, meaning dynamic or noisy environments or highly nonlinear problems, type-2 fuzzy systems have shown that can outperform type-1 fuzzy systems. In addition, as another extension of type-1, intuitionistic fuzzy systems (IFSs) emerged as another way to model uncertainty in real world problems. Even with all of these previous works, an open question still remains, which is, if type- 1 or type- 2 fuzzy systems are sufficient to model all existing problems in the real world.

The main contribution of this paper is a proposed mathematical framework to find the optimal fuzzy system (type-1, type-2 or intuitionistic) for a particular problem or class of problems, in the sense that the fuzzy model should approximate in the best way possible the real dynamical system. Of course, the proposed approach is posed as an optimization problem because we need to minimize the error in some particular metric measuring the difference between the outputs of the fuzzy model and the real dynamical system under consideration.

The rest of the paper is organized as follows. Section 2 outlines some related works on optimization of fuzzy systems and granularity. Section 3 outlines the proposed method for finding the optimal fuzzy system for a particular application. Section 4 describes an implementation approach of the proposed method using genetic algorithms. Finally, Section 5 offers some conclusions and possible future works in this area.

\section{Related works}

As related works we can mention papers that are related to type- $n$ and intuitionistic fuzzy systems, but from the granularity based approach, like in the works of Witold Pedrycz that we briefly describe below. First, there is the work by Pedrycz [17] on Algorithmic Developments of Information Granules of Higher Type and Higher Order and Their Applications in which it is described very clearly how type-n information granules are used in the augmentation of numeric models. Another work by Pedrycz [15] is on the development of granular metastructures and their use in a multifaceted representation of data and models presents a constructive way of forming type- 2 fuzzy sets via the principle of justifiable granularity exhibits a significant level of originality and offers a general way of designing information granules. In addition, the work by Pedrycz [16] on Hierarchical Architectures of Fuzzy Models: From Type-1 fuzzy sets to Information Granules of Higher Type, which describes the 
enhanced interpretability of fuzzy sets by elaborating on the role of type- 2 fuzzy sets (which offers an effective vehicle of linguistic quantification of numeric membership degrees) and shadowed sets (with their ability to express uncertainty). Finally, also in the work by Pedrycz [18] on Concepts and Design Aspects of Granular Models of Type-1 and Type-2 some interesting ideas on forming type 2 fuzzy models are presented.

There also several recent works on developing type-2 fuzzy models in diverse areas of application that can be viewed as related work. In the work by Melin et al. [11] on Edgedetection method for image processing based on generalized type-2 fuzzy logic, an approach using type-2 fuzzy for edge detection that outperforms other methods is presented. In the work of Rubio et al. [19] an Extension of the Fuzzy Possibilistic Clustering Algorithm using Type-2 Fuzzy Logic Techniques is presented. In the work of Olivas et al. [14] a Comparative Study of Type-2 Fuzzy Particle Swarm, Bee Colony and Bat Algorithms in Optimization of Fuzzy Controllers is outlined. In the work of Castillo et al. [6] a Review of Recent Type-2 Fuzzy Image Processing Applications is presented. In the work of Gonzalez et al. [9] an optimization method of interval type-2 fuzzy systems for image edge detection is described. In the work of Tai et al. [22] a Review of Recent Type-2 Fuzzy Controller Applications is presented. In the work of Sepulveda et al. [21] an Experimental study of intelligent controllers under uncertainty using type-1 and type-2 fuzzy logic is described. In the work of Castillo and Melin [7] the Design of Intelligent Systems with Interval Type-2 Fuzzy Logic is presented, and this work includes the Theory and Applications of the design process. Finally, in Sanchez et al. [20] Generalized Type-2 Fuzzy Systems for controlling a mobile robot and a performance comparison with Interval Type-2 and Type-1 Fuzzy Systems are presented.

\section{Proposed method}

Initially, we can have fuzzy models of type-1 that can be represented as follows:

$$
\begin{gathered}
A: X \rightarrow[0,1] \\
X=\left\{x_{1}, x_{2}, \ldots, x_{n}\right\},
\end{gathered}
$$

where $A$ is the so called membership function, and $X$ is the domain of interest.

Then these models can be extended by considering uncertainty in the membership functions, in this way obtaining type-2 fuzzy models as follows:

$$
\begin{gathered}
A: X \rightarrow F([0,1]) \\
X=\left\{x_{1}, x_{2}, \ldots, x_{n}\right\},
\end{gathered}
$$

where $F$ represents families of type- 1 fuzzy sets, and in this case the interval type- 2 fuzzy models are also included.

In the same way, we can extend the fuzzy models once more by considering uncertainty in the type-2 fuzzy models, in this way obtaining type-3 fuzzy models as follows:

$$
\begin{aligned}
& A: X \rightarrow F^{2}([0,1]) \\
& X=\left\{x_{1}, x_{2}, \ldots, x_{n}\right\},
\end{aligned}
$$

where $F^{2}$ stands in this case for families of type-2 fuzzy sets. 
For the case of intuitionistic fuzzy models, the uncertainty is represented by a membership and non-membership function, so the representation is as follows:

$$
A: X \rightarrow F([0,1],[0,1])
$$

It is also possible that intuitionistic type- 2 fuzzy models can be represented as follows:

$$
A: X \rightarrow F^{2}([0,1],[0,1])
$$

Now we can pose the problem of designing a fuzzy system that models the uncertainty represented by $\varepsilon$ as the maximization of data coverage as follows:

$$
\begin{aligned}
& \operatorname{Max}_{\varepsilon} \sum_{k=1}^{N} \text { coverage(target data) } \\
& \text { such that } \\
& \sum_{i=1}^{p} \varepsilon_{i}=\varepsilon \text { and } \varepsilon_{i} \geq 0 \text {, }
\end{aligned}
$$

where "target data" means available input-output training data to construct the model, $\mathrm{N}$ means the number of data points, $p$ is the number of fuzzy sets used (depending on the performed granulation). The meaning of "coverage" is defined by the ratio of data points covered by the fuzzy model out of the total of points, which is at most one for a perfect model.

The concept of coverage of a fuzzy set, $\operatorname{cov}($.$) is discussed with regards to some$ experimental data existing in $R^{n}$, that is $\left\{x_{1}, x_{2}, \ldots, x_{N}\right\}$. As the name itself stipulates, coverage is concerned with an ability of a fuzzy set to represent (cover) these data. In general, the larger number of data is being "covered", the higher the coverage of the fuzzy set. Formally, the coverage can be sought as a non-decreasing function of the number of data that are represented by the given fuzzy set A. The monotonicity property of the coverage measure is obvious: the higher the values of $\varepsilon$, the higher the resulting coverage. Hence the coverage is a nondecreasing function of $\varepsilon$.

The problem can be re-structured in the following form in which the objective function is a product of the coverage and specificity-determine optimal allocation of information granularity $\left[\varepsilon_{1}, \varepsilon_{2}, \ldots, \varepsilon_{p}\right]$ so that the coverage and specificity criteria become maximized.

Finally, we can pose the general optimization of the fuzzy model, meaning finding the appropriate value of $n$ for a fuzzy model according to the target data of the problem. In this case, it is a minimization problem that can be stated in general as follows:

$$
\operatorname{Min}_{n}\left\|F^{n}(M)-R S\right\|<\tau,
$$

where $\tau$ is accuracy threshold, which is application related. In this case, the Euclidean distance can be used as the norm, but others could be used depending on the application area. The minimization problem can be solved by any optimization method in the literature, although due to the complexity issue we prefer to use meta-heuristic algorithms that can provide a sufficiently good approximation to the optimal solution of the problem.

We envision that different $n$ values would be the optimal ones for certain classes of problems depending on the complexities and nonlinearities.

For the moment, we can solve this problem by using bio-inspired or meta-heuristic optimization algorithms due to the high computational overhead required in this hierarchical optimization problem. 


\section{Intuitionistic fuzzy logic systems}

According to Atanassov [1-5], an IFS on the universum $X \neq \varnothing$ is an expression $A$ given by:

$$
A=\left\{\left\langle x, \mu_{A}(x), v_{A}(x)\right\rangle \mid x \in X\right\},
$$

where the functions

$$
\mu_{A}, v_{A}: X \rightarrow[0,1]
$$

satisfy the condition

$$
0 \leq \mu_{A}(x)+v_{A}(x) \leq 1
$$

and describe, respectively, the degree of the membership $\mu_{A}(x)$ and the non-membership $v_{A}(x)$ of an element $x$ to $A$. Let

$$
\pi_{A}(x)=1-\mu_{A}(x)-v_{A}(x)
$$

therefore, function $\pi_{A}$ determines the degree of uncertainty.

According to [5] the geometrical forms of the intuitionistic fuzzy numbers can be generalized as follows: For the first case functions $\mu_{A}$ and $v_{A}$ satisfied the conditions [5]:

$$
\sup _{y \in E} \mu_{A}(y)=\mu_{A}(x)=a, \inf _{y \in E} v_{A}(y)=v_{A}(x)=b,
$$

for each $x \in\left[x_{1}, x_{2}\right]$, and for the second case [5]:

$$
\sup _{y \in E} \mu_{A}(y)=\mu_{A}\left(x_{0}\right)=a, \inf _{y \in E} v_{A}(y)=v_{A}\left(x_{0}\right)=b .
$$

For the first case we have:

$\mu_{A}$ is increasing function from $-\infty$ to $x_{1}$;

$\mu_{A}$ is decreasing function from $x_{2}$ to $+\infty$;

$v_{A}$ is decreasing function from $-\infty$ to $x_{1}$;

$v_{A}$ is increasing function from $x_{2}$ to $+\infty$.
For the second case we have:

$\mu_{A}$ is increasing function from $-\infty$ to $x_{0}$;

$\mu_{A}$ is decreasing function from $x_{0}$ to $+\infty$;

$v_{A}$ is decreasing function from $-\infty$ to $x_{0}$;

$v_{A}$ is increasing function from $x_{0}$ to $+\infty$.

Obviously, in both cases the functions $\mu_{A}$ and $v_{A}$ can be represented in the form

$$
\mu_{A}=\mu_{A}^{\text {left }} \cup \mu_{A}^{\text {right }}, v_{A}=v_{A}^{\text {left }} \cup v_{A}^{\text {right }},
$$

where $\mu_{A}^{\text {left }}$ and $v_{A}^{\text {left }}$ are the left, while $\mu_{A}^{\text {right }}$ and $v_{A}^{\text {right }}$ are the right sides of these functions.

Therefore, the above conditions can be re-written in the (joint) form [5]:

$$
\sup _{y \in E} \mu_{A}(y)=\mu_{A}(x)=a, \quad \inf _{y \in E} v_{A}(y)=v_{A}(x)=b,
$$

for each $x \in\left[x_{1}, x_{2}\right]$ and in the particular case, when $x_{1}=x_{0}=x_{2}, \mu_{A}^{\text {left }}$ is increasing function; $\mu_{A}^{\text {right }}$ is decreasing function; $v_{A}^{\text {left }}$ is decreasing function and $v_{A}^{\text {right }}$ is increasing function. Following [5], we will consider, ordered by generality, the definitions:

1. In the graphical representation in both cases above $a=1, b=0$.

2. $\sup _{y \in E} \mu_{A}(y)=\mu_{A}\left(x_{0}\right)>0.5>v_{A}\left(x_{0}\right)=\inf _{y \in E} v_{A}(y)$. 

3. $\sup _{y \in E} \mu_{A}(y)=\mu_{A}\left(x_{0}\right) \geq 0.5 \geq v_{A}\left(x_{0}\right)=\inf _{y \in E} v_{A}(y)$.
4. $\sup _{y \in E} \mu_{A}(y)=\mu_{A}\left(x_{0}\right)>v_{A}\left(x_{0}\right)=\inf _{y \in E} v_{A}(y)$.
5. $\sup _{y \in E} \mu_{A}(y)=\mu_{A}\left(x_{0}\right) \geq v_{A}\left(x_{0}\right)=\inf _{y \in E} v_{A}(y)$.
6. $\sup _{y \in E} \mu_{A}(y)=\mu_{A}\left(x_{0}\right)>0$.
7. $\inf _{y \in E}=v_{A}\left(x_{0}\right)<1$.

This is just a brief description of the extensive work on this area of Prof. Atanassov.

\section{Proposed implementation of the method}

For illustration purposes we consider in this section how the proposed method can be implemented for finding the optimal fuzzy systems. The general idea can easily be extended to type- $n$ intuitionistic by increasing the corresponding size of the chromosome. We can use for this implementation a genetic algorithm to perform the above mentioned optimization process.

The chromosome of the proposed hierarchical genetic algorithm for the fuzzy inference system optimization is shown in Figure 1, where control genes allow determining the type of fuzzy logic, type of system and number of membership functions for each variable (inputs and outputs) and depending of their values, the rest of the genes are activated.

This proposed approach for the implementation can be easily extended to consider higher types for the fuzzy models (not just type- 1 and type- 2 or intuitionistic) in a similar fashion as illustrated in this section. The optimization method could be changed, for example using particle swarm optimization or other similar meta-heuristic, but in general the design process is the same.

\section{Conclusions}

In this paper a method for finding the optimal fuzzy systems was presented. In addition, a framework for solving this problem of finding the optimal type-1, type-2 or intuitionistic fuzzy model was presented. We envision that in the future even more general intuitionistic type- $n$ fuzzy models will be used more frequently for solving complex problems, as real world situations are becoming more complicated by dynamic and non-linear environments, as well as huge amounts of data being available for processing very quickly in real time decision making. In particular, we expect to test the proposed approach with problems of nonlinear control, time series prediction, and pattern recognition. As future work, theoretical as well as applied works are envisioned that will be done, as this paper is just the initial and very modest work being done in this area. 


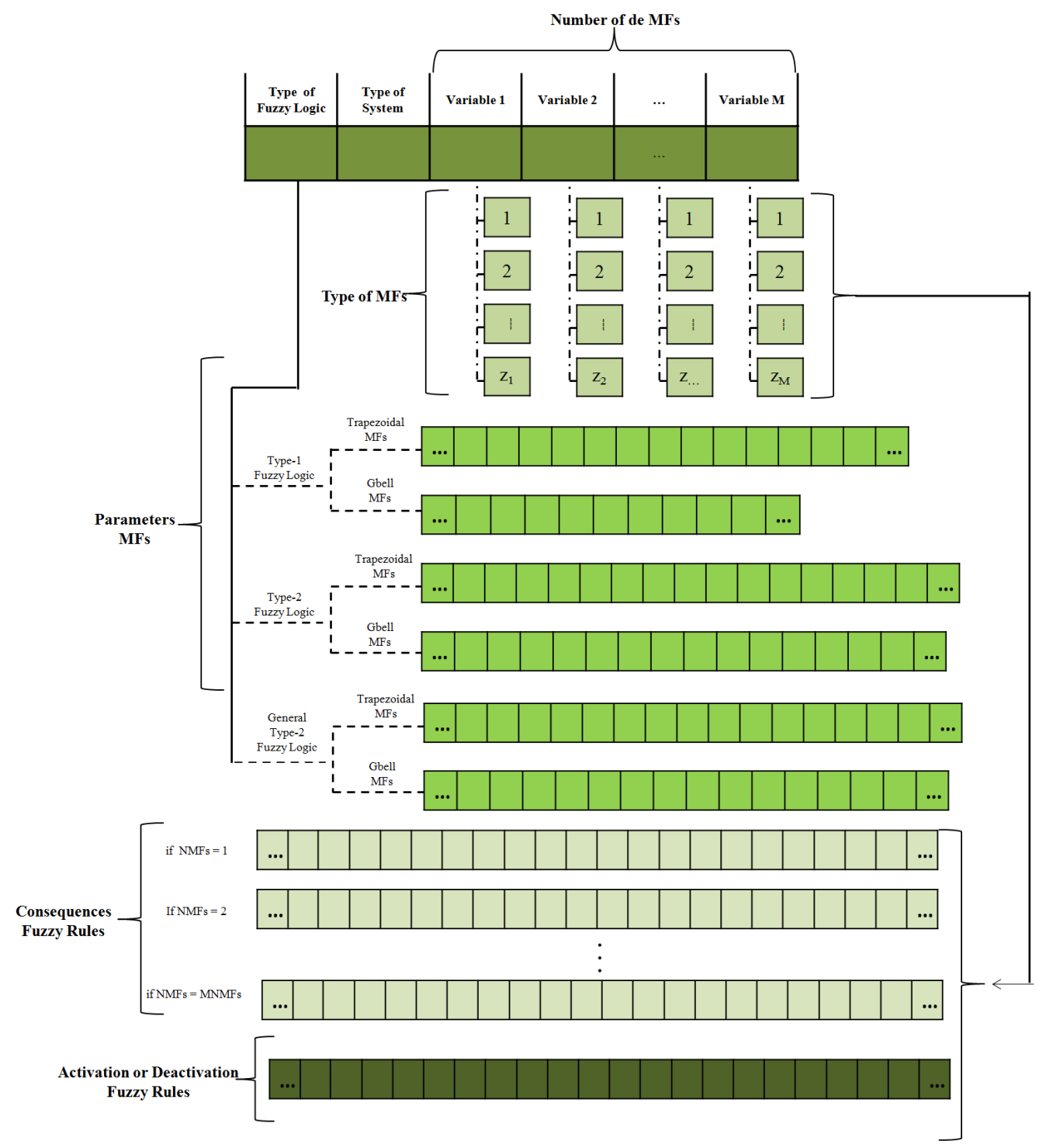

Figure 1. Chromosome of the proposed HGA for optimal design of fuzzy systems

\section{References}

[1] Atanassov, K. (1986) Intuitionistic fuzzy sets, Fuzzy Set and Systems, 20(1), 87-96.

[2] Atanassov, K. (1983) Intuitionistic fuzzy sets, VII ITKR Session, Sofia, 20-23 June 1983, Reprinted: Int. J. Bioautomation, 20(S1), 2016, S1-S6.

[3] Atanassov, K. (1999) Intuitionistic Fuzzy Sets: Theory and Applications, Springer, Heidelberg.

[4] Atanassov, K. (2012) On Intuitionistic Fuzzy Sets Theory, Springer, Berlin.

[5] Atanassov, K., Vassilev, P. \& Tsvetkov, R. (2013) Intuitionistic Fuzzy Sets, Measures and Integrals. Academic Publishing House "Prof. Marin Drinov", Sofia.

[6] Castillo, O., Sanchez, M. A., González, C. I. \& Martinez, G. E. (2017) Review of Recent Type-2 Fuzzy Image Processing Applications. Information 8(3), 97, (18 pages). 
[7] Castillo, O, \& Melin, P. Design of Intelligent Systems with Interval Type-2 Fuzzy Logic, Type-2 Fuzzy Logic: Theory and Applications, 53-76.

[8] Castillo, O., Melin, P., Ramírez, E., \& Soria, J. (2012) Hybrid intelligent system for cardiac arrhythmia classification with Fuzzy K-Nearest Neighbors and neural networks combined with a fuzzy system, Expert Systems with Applications 39 (3), 2947-2955.

[9] González, C. I., Melin, P., Castro, J. R., Castillo, O. \& Mendoza, O. (2016) Optimization of interval type-2 fuzzy systems for image edge detection. Appl. Soft. Comput., 47, 631643

[10] Melin, P. \& Castillo, O. (2001) Modelling, simulation and control of non-linear dynamical systems: an intelligent approach using soft computing and fractal theory, CRC Press.

[11] Melin, P., Gonzalez, C. I., Castro, J. R., Mendoza, O. \& Castillo, O. (2014) Edgedetection method for image processing based on generalized type-2 fuzzy logic, IEEE Transactions on Fuzzy Systems, 22(6), 1515-1525.

[12] Melin, P. \& Castillo, O. (2001) Intelligent control of complex electrochemical systems with a neuro-fuzzy-genetic approach, IEEE Transactions on Industrial Electronics, 48(5), 951-955.

[13] Mendez, G. M., \& Castillo, O. (2005, May). Interval type-2 TSK fuzzy logic systems using hybrid learning algorithm, Fuzzy Systems, 2005. FUZZ'05. The 14th IEEE International Conference on, 230-235.

[14] Olivas, F., Amador-Angulo, L., Perez, J., Caraveo, C., Valdez, F., \& Castillo, O. (2017). Comparative Study of Type-2 Fuzzy Particle Swarm, Bee Colony and Bat Algorithms in Optimization of Fuzzy Controllers. Algorithms, 10(3), 101 (27 pages).

[15] Pedrycz, W. (2010). The development of granular metastructures and their use in a multifaceted representation of data and models. Kybernetes, 39(7), 1184-1200.

[16] Pedrycz, W. (2010). Hierarchical architectures of fuzzy models: From type-1 fuzzy sets to information granules of higher type. International Journal of Computational Intelligence Systems, 3(2), 202-214.

[17] Pedrycz, W. (2016) Algorithmic Developments of Information Granules of Higher Type and Higher Order and Their Applications. In: International Workshop on Fuzzy Logic and Applications, 27-41.

[18] Pedrycz, W. (2015) Concepts and Design Aspects of Granular Models of Type-1 and Type-2. Int. J. Fuzzy Logic and Intelligent Systems, 15(2), 87-95.

[19] Rubio, E., Castillo, O., Valdez, F., Melin, P., Gonzalez, C. I., \& Martinez, G. (2017). An Extension of the Fuzzy Possibilistic Clustering Algorithm Using Type-2 Fuzzy Logic Techniques. Adv. Fuzzy Systems, 2017, Article ID 7094046, 23 pages.

[20] Sanchez, M. A., Castillo, O., \& Castro, J. R. (2015). Generalized type-2 fuzzy systems for controlling a mobile robot and a performance comparison with interval type-2 and type-1 fuzzy systems. Expert Systems with Applications, 42(14), 5904-5914. 
[21] Sepúlveda, R., Castillo, O., Melin, P., Rodríguez-Díaz, A., \& Montiel, O. (2007). Experimental study of intelligent controllers under uncertainty using type-1 and type-2 fuzzy logic. Information Sciences, 177(10), 2023-2048.

[22] Tai, K., El-Sayed, A. R., Biglarbegian, M., Gonzalez, C. I., Castillo, O., \& Mahmud, S. (2016). Review of recent type-2 fuzzy controller applications. Algorithms, 9(2), 39 (19 pages). 